\title{
FSU: ZWISCHEN MÜNDLICHKEIT UND SCHRIFTLICHKEIT
}

\author{
BARBARA SKOWRONEK \\ ALEKSANDRA PUTOWSKA
}

1. Menschen brauchten schon immer Fremdsprachenkenntnisse, ob für allgemein-bildende und gesellschaftlich-kommunikative Zwecke, um schriftlich und/oder mündlich zu verstehen und verstanden zu werden, um andere Menschen zu kontaktieren, um andere Länder und Kulturen kennen zu lernen, privat und/oder beruflich und fachorientiert. Dazu eignen sich zwei Zugangswege:

- der natürliche Weg der wirklichen zwischenmenschlichen Kontakte, durch Interaktion des Lerners mit seiner Umgebung (direkte Methode)

- und der institutionell gesteuerte Weg, begonnen mit dem Unterricht Alter Sprachen (Griechisch und Latein) nach der Grammatik-Übersetzungs-Methode als dem bildenden Vorrang (indirekte Methode).

$\mathrm{Zu}$ unterscheiden sind also zwei Hauptrichtungen des (Fremd)Sprachen-Erlernens: der direkte Weg (natürlich, oft auf kommunikativer Mündlichkeit basierend, also etwa so, wie Kleinkinder ihre erste Sprache erwerben) und der indirekte Weg (gesteuert, auf Schriftlichkeit in Grammatik und Übersetzung basierend).

Der FSU ist institutionell, schulisch gesteuert, daher muss er sich nach bestimmten Grundsätzen richten, die mit Hilfe von bestimmten Methoden realisiert werden. Methoden im FSU sind als Wegweiser und Verfahrensweisen für das Erreichen von beabsichtigten Unterrichtszielen, gestützt auf wissenschaftliche Prinzipien, zu verstehen.

Der FSU verläuft in Bildungsinstitutionen fremdbestimmt und gesteuert; es gibt den Lehrer, der portionierten Lerngegenstand an seine Lerner zu vermitteln bekommt (und hat), in bestimmte Reihenfolgen, bestimmten Zeitverlauf und Auswahl 
gebracht und verteilt. Es gibt auch Experten für das gesteuerte Lernen, die eine Unterteilung der Zielsprache als Lerngegenstand vornehmen. All das geschieht gesteuert, „künstlich“; auch die differenzierende Behandlung der gesprochenen und der geschriebenen Sprache erfolgt im FSU gesteuert. (vgl. Rösler, 2016: 136).

Indirekte Methoden werden (hauptsächlich) vom Lehrer mit Hilfe von Vermittlungssprache(n) (oft der Muttersprache) im geplanten, gesteuerten FSU realisiert; dieser Unterricht verläuft nur (hauptsächlich) auf die Vermittlung von Sprachwissen konzentriert, um zu lernen, vorgegebene grammatisch korrekte Sätze zu verstehen, zu bilden und zu übersetzen; angestrebt wird nur korrektes Regelwissen über das Sprachsystem.

Der traditionelle FSU, hauptsächlich nach der Grammatik-Übersetzungs-Methode war lehr(er)orientiert: die wichtigste Rolle (Primärstellung) kam den Unterrichtsprozessen und den unterrichtenden Personen (Lehrern) zu:

- der Lehrer war aktives Unterrichtssubjekt, der zu entscheiden hatte, wie muss/ wie soll der Unterricht konzipiert, geplant, organisiert, durchgeführt werden, damit das Unterrichtsergebnis (bspw. Noten) zufriedenstellend ist;

- die Aufgabe des Lerners war, sich noten- und niveaugerecht dem Lehrer unterzuordnen und auf die erwünschten (erwarteten) Resultate hin zu lernen. Der Lerner war passives Unterrichtsobjekt, auf Wissensempfang vom Lehrer ausgerichtet. Vom Lerner wurde nur korrektes, fehlerfreies Schreiben und Sprechen, aufgebaut auf korrektem Sprachwissen (nur) zu vorgegebenen, grammatisch profilierten Themen erwartet; tatsächliches spontanes Reden in wirklichen Kommunikationssituationen war nicht erwartet, fast unerwünscht, weil potentiell fehlerhaft.

2. In Polen beschäftigt man sich mit Spracherwerb, Sprachenlernen, Sprachvermittlung innerhalb der Glottodidaktik, einer wissenschaftlichen Disziplin, die dank Prof. Ludwik Zabrocki (1907-1977) innerhalb der 1964 entstandenen Sektion für Angewandte Sprachwissenschaft an der Adam-Mickiewicz-Universität in Poznań gegründet wurde (damit hat sich die institutionelle Verselbständigung der Angewandten Sprachwissenschaft in Polen vollzogen). 1966 wurde (ebenso dank L. Zabrocki) die erste Nummer der internationalen Zeitschrift Glottodidactica zum Gedankenaustausch für angewandt-linguistische Forschungen herausgegeben (die bis heute erfolgreich herausgegeben wird).

Glottodidaktik ist eine integrative menschenbezogene Disziplin zur wissenschaftlichen und praktischen Erforschung der Vermittlung natürlicher Sprachen, sowohl im gesteuerten institutionellen FSU (unter schulischen Bedingungen) als auch im natürlichen Erstspracherwerb und Zweitspracherwerb. Der Untersuchungsgegenstand der Glottodidaktik (großteils) ist der FSU, genauer gesagt Sprache als Kommunikationsmittel und Unterrichtsgegenstand in verschiedenen Konstellationen 
des glottodidaktischen Gefüges (Lerner, Lehrer, Sprache(n)). Wichtiges Objekt glottodidaktischer Untersuchungen ist Sprache im (und für den) FSU.

Der institutionelle FSU durchlief mehrere Methoden, von denen manche noch bis heute praktiziert werden. Einfluss auf den Methodenwandel innerhalb des FSU haben Entwicklungen in der Linguistik.

3. Sprache ist das primäre Untersuchungsobjekt der Linguistik, innerhalb deren man sich mit verschiedenen Aspekten von „Was ist Sprache?”, „Wie funktioniert Sprache?" beschäftigt. Daher sind Entwicklungen in der Linguistik glottodidaktisch relevant; das heißt in glottodidaktischen Untersuchungen sollte man sich auf linguistische Untersuchungen stützen. Will man der Frage nach dem „Wie und was geschieht im FSU?” nachgehen, muss zuerst auf die Frage „Was ist und wie funkioniert Sprache?" auch im geschichtlichen Methodenwandel geantwortet werden. Glottodidaktische Konzeptionen sind linguistisch zu begründen.

4. Bis zum Ende des 19. Jh. hatte man sich wissenschaftlich mit Sprache jahrhundertelang und Europa-weit innerhalb der Alt-Philologie befasst, mit dem Hauptziel der Rekonstruktion literarischer Texte über das Leben und die Gesellschaftsordnung der Alten Griechen und Römer, um Erkenntnisse über deren allumfassende Lebensweisen zu gewinnen (Grucza, 1988: 34). Die damaligen sprachwissenschaftlichen Studien waren diachronisch: historisch-vergleichend, auf Etymologie aufgebaut. Die Alten Zeiten hielt man für klassisches Muster für die Gegenwart und Zukunft, Latein glaubte man als Muster logischen Denkens. Der Schwerpunkt des wissenschaftlichen Interesses der damaligen Alt-Philologen waren originale Schrifttexte als Dokumente der Vergangenheit. Die damalige Linguistik war textbezogen, autonom: vom Menschen und von Alltagskultur getrennt (Grucza, 2007: 377), im Vordergrund stand die Hochsprache (und Hochkultur).

Altgriechisch und Latein waren jahrhundertelang hoch geschätzte Bildungssprachen der gebildeten Eliten in Europa und gehören bis heute zum kulturellen Erbe Europas. Unterrichtet wurden sie für rein bildende Zwecke in meistens konfessionellen Elite-Schulen für Jungen mit den Schwerpunkten: Schriftlichkeit in Grammatik und Übersetzung (nach der Grammatik-Übersetzungs-Methode). Da es keine andere Methode für den institutionellen FSU gab, wurden auch die sog. Neuen Sprachen (Volkssprachen, Nationalsprachen) als Fremdsprachen institutionell ebenso nach der G-Ü-M unterrichtet.

An der Wende des 19. Jahrhunderts zum Beginn des 20. Jahrhunderts kam es mit der institutionellen Akzeptanz der Phonologie (1850) zur akademischen Verselbständigung der Sprachwissenschaft: die Neuphilologie war geboren! Der Bedeutungsumfang der Neuphilologie erweiterte sich auf jede beliebige Sprache, Kultur, Literatur, also auf Nationalsprachen (aus den sog. Vulgärsprachen) (Grucza, 1988: 32). 
In Polen war es Prof. A. Kleczkowski (1888-1949) der an der Jagiellonen-Universität Kraków schon vor dem Zweiten Weltkrieg die bahnbrechende Notwendigkeit erkannte, vergleichende deutsch-polnische kontrastive gegenwartsbezogene sprach- und literaturwissenschaftliche Studien zu betreiben.

Später bestimmte Prof. Ludwik Zabrocki an der Adam-Mickiewicz-Universität in Poznań das Ziel der Neuphilologie an polnischen Universitäten, Wissenschaftler zur Literatur und Sprache auszubilden. Folglich wurde im Rahmen des neuphilologischen Studiums eine gesonderte Fachrichtung zur Ausbildung von Lehrkräften gegründet; die dazu notwendigen Studienprogramme für Germanistik-Studenten wurden ebenso von Zabrocki erarbeitet $(1962,1967)$.

5. Ebenso an der Wende des 19. zum Beginn des 20. Jahrhunderts herrschte ein weltweites wirtschaftliches Chaos, das zum finanziellen Ruin, Unruhen und Unsicherheit bei vielen Menschen führte, die folglich in der Suche nach Arbeit nach Amerika auswandern mussten. Migrationsbewegungen der Menschen verursachten wiederum das dringende Bedürfnis nach neuen Fremdsprachenkenntnissen. Demzufolge blieb die Zeit auch am FSU nicht spurlos stehen: es mussten sehr bald Änderungen erfolgen, den damaligen Migrations-Entwicklungen angepasst. Zwar war der Unterricht nach der Grammatik-Übersetzungs-Methode (mit dem Schwerpunkt auf Schriftlichkeit) für den Unterricht Alter Sprachen auch für den Unterricht Neuer Sprachen adaptiert; aber auf Dauer erwies sich diese Methode für den Unterricht Neuer Sprachen (die zunehmend Mündlichkeit brauchten) als völlig unzureichend.

5.1. In Europa war es Viëtor, der sich 1882 mit seinem Aufsatz Der Sprachunterricht muss umkehren im Rahmen der sog. Reformbewegung zum ersten Mal theoretisch der Grammatik-Übersetzungs-Methode widersetzte (geprägt durch Überlegungen zum Erstspracherwerb von Kleinkindern von Gouïn, 1880).

Viëtor kritisierte die bislang geltende Grammatik-Übersetzungs-Methode als untauglich für den Unterricht Neuer Sprachen, weil diese Methode hauptsächlich zum Erlangen des theoretischen und schriftlichen Sprachwissens (als auswendig gelernte grammatische Paradigmen-Reihen mitsamt den Ausnahmen) beitrage, nicht zum tatsächlich brauchbaren mündlichen Sprachkönnen im Alltag.

So kam es, dass sich die direkte Methode (mit Druck auf Mündlichkeit) für den FSU Neuer Sprachen in Europa entwickelte und institutionell ausbreitete und als offiziell anerkannte Reform-Methode ihre öffentliche Anwendung in Schulen fand.

Direkte Methoden (manchmal auch Gouvernanten-Methoden genannt) ergeben sich aus der Beobachtung des natürlichen Erstspracherwerbs, wie Mütter bei ihren Kleinkindern das Aneignen der Sprache lenken: durch bloßes spontanes kommunikatives Erproben in Alltagssituationen, ohne grammatische Steuerung, als Spracheim-Gebrauch. Kleinkinder erwerben Sprache aus der Interaktion mit ihrer Umgebung, die durch eine bestimmte dialektale oder soziale Variante geprägt ist: ob 
Hochsprache oder Dialekt, ob Jugendsprache oder Oma-Sprache usw. - das übernimmt das Kleinkind.

Dies ist die natürlichste Art, Sprache in der realen, tatsächlichen Sprachumgebung eingetaucht (immersiv) zu lernen, nur auf kommunikatives (sprachlich-kulturelles) Können (lernerzentriert) ausgerichtet, lässt sich auch in den FSU einbinden.

Sprachliche und nichtsprachliche Selbstversuche der Kleinkinder und Lerner, die Sprache und Wissen in ihrer Umgebung erlernen, werden eigentlich für außersprachliche Verständigungs-Zwecke unternommen: um zu verstehen und verstanden zu werden. Das Kleinkind, welches Sprache erwirbt, versucht, die gesamte Kommunikationssituation zu begreifen, zu verstehen und sich verständlich zu machen; zuerst nur mit dem ganzen Körper: so wird nicht nur die Mobilität des Kindes geübt, sondern der Sprachrhythmus und Sprachklang der Umgebung nachvollzogen, später zunehmend sprachlich. Lerner benutzen am Anfang des Sprachen-Lernens viele feste Kommunikationsformeln, reichlich durch nonverbale Kommunikationsmittel (,mit Händen und Füßen”) unterstützt.

Die direkte Methode wurde auch für die Kommunikation mit Muttersprachlern benutzt: entweder im fremdsprachlichen Ausland, oder für die Kommunikation mit Gouvernanten, die in reichen Häusern angestellt waren, um Kindern Fremdsprachen natürlich zu vermitteln (sie „besprachen” ihre Schüler nur mit ihrer Muttersprache, da sie die Sprache der Kinder (Schüler) nicht kannten). Diese Methode wurde also in natürlichen Kontakten zwischen Menschen verschiedener Sprachen verwendet, sowie im häuslichen FSU; ihr Ziel war hauptsächlich das Erreichen des mündlichen Sprachgebrauchs nach dem Sprachmuster der Lehrperson. Auf den Prinzipien der direkten Methode basiert man auch heute noch.

5.2. Auch frühere Menschenwanderungen in Europa kreuz und quer erforderten gute kulturdeterminierte Sprachkenntnisse zur Verständigung mit Einheimischen. Also zeigte sich die Notwendigkeit, Fremdsprachen in kulturdeterminierten Relationen zu erlernen und zu unterrichten. Unter Reisenden waren viele Studenten, die sich auf Studienreisen Europa-weit begaben und Sprachen durch kommunikative Kontakte für Studien-, Handels- und Kulturzwecke erlernten. Der einfachste und natürlichste Weg war (und ist es bis heute), eingetaucht in die fremdsprachliche Umgebung, sozusagen immersiv, spontan (sprachlich und nichtsprachlich) hauptsächlich mündlich zu kommunizieren zu versuchen. Wahre Kommunikation in der Umgebung ist der direkte Weg, Sprache zu erlernen und sich an der Verständigung zu erproben: so, wie es Kleinkinder tun, wenn sie unbewusst interagieren und kommunikativ (irgend)eine (Erst-/Mutter-)Sprache erwerben: beobachtend zuhören, nachahmen, um sich auch selbst sprachlich und nichtsprachlich kommunikativ zu versuchen. Wie sehr gute Beobachter Kinder sind, hat so manchen Erwachsenen ins Staunen versetzt und zum Lernen angespornt. 
Ähnlich erwerben Erwachsene, die im Ausland Sprache und Weltwissen aus der kommunikativen Interaktion mit der Umgebung erlernen, eine bestimmte regionale und soziale Variante der Sprache, die durch historisches und kulturelles Wissen determiniert ist; dies geschieht meistens umgebungs- und interessengeleitet. (Rösler, 2016: 136).

5.3. In Amerika war es Berlitz, der sehr bald die Zeichen der Zeit erkannte, und seit 1878 Sprach-Schulen für Europa-Einwanderer, die in Amerika nach Arbeit suchten, also Sprachkurse mit Englisch als Fremdsprache organisierte, hauptsächlich in New York und anderen Orten der USA an der Atlantik-Küste. Zu diesem Zweck engagierte er Englisch sprechende Muttersprachler, die den FSU konsequent direkt als Muttersprache-Englisch realisierten. Den Neuankömmlingen wurde eine Menge brauchbarer kommunikativer (hauptsächlich mündlicher) Text-und-Satzmuster für den alltäglichen Anfänger-Unterricht vermittelt, der ihnen Neuorientierung und Neubeginn, auch in Ämtern ermöglichte.

Dringend gebraucht wurden Kenntnisse der wirklichen Gegenwartssprache, Alltagssprache, um notwendige, aktuelle altägliche Besorgungen im Ausland zu machen, um zu überleben (nicht eine klassische Hochsprache nach dem Muster der Antike, auch nicht die bspw. englische Elite-Hochsprache, Standard-Sprache).

Das heißt, gefragt war und intensiv geübt wurde konzeptionelle Mündlichkeit zeitgleich mit der medialen Mündlichkeit, also Gesprächsmuster für den wirklichen kommunikativen situationsadäquaten Gebrauch, möglichst Aussprache-korrekt.

Auch wurde deutlich, dass Sprache kulturgeprägt zu vermitteln ist. Migranten merkten sehr bald kulturelle Andersartigkeiten in Amerika, die im Sprachgebrauch ihren Niederschlag fanden und die ebenso akzeptiert oder zumindest respektiert werden mussten: neben praktischem Sprachwissen und -können musste also praktisches Kulturwissen und -können integrativ in den FSU eingebunden werden (nicht wie früher Sprache und Kultur voneinander getrennt, als autonome Einheiten nebeneinander).

An der Wende vom 19. zum 20. Jahrhundert erfolgte auch die Entwicklung des institutionell gesteuerten FSU vom Erlernen grammatisch korrekter schriftlicher Texte (nach der G-Ü-Methode) zum tatsächlichen kommunikativen (vorrangig mündlichen) Sprachgebrauch (nach der direkten Reform-Methode). Das Ziel wurde von der Norm-Grammatik-Korrektheit auf die alltägliche, intuitiv anwendbaren Gebrauchs-Grammatik verschoben: ausschlaggebend sollte nun sein: (v.a.) mündlich verstehen und verstanden werden. Folglich mussten die notwendigen Sprachfertigkeiten im FSU primär vom Leseverstehen und Schreiben auf Hörverstehen (Ohr) und Sprechen (Mund), also von Schriftlichkeit auf Mündlichkeit verschoben und geübt werden.

5.4. Wahrscheinlich verursachte die Weltwirtschaftskrise dieser Zeit, dass der FSU zunehmend gebrauchsorientiert und mündlich wurde; sie wurde auch der Aus- 
löser für die zeitgleiche Verschiebung des wissenschaftlichen Interesses innerhalb der Linguistik von etymologischen historisch-diachronischen Studien alter Texte auf gegenwartsorientierte Sprache-im-Gebrauch. Demzufolge kam es zur institutionellen Verselbständigung der Sprachwissenschaft (dank Phonologie) und zur Entstehung der Neuphilologie.

Das Entwicklungs-Pendel der Linguistik und des FSU schlug von dem Interesse für primär schriftliches Sprachwissen der Hochsprachen im Leseverstehen und Schreiben zum mündlichen alltäglichen Sprachgebrauch, also von Schriftlichkeit zur Mündlichkeit.

6. Die strukturalistische Wende prägte den FSU. Dank Ferdinand de Saussure (gest. 1916) kam es zur strukturalistischen Wende in der Linguistik: in seinen sprachwissenschaftlichen Vorlesungen an der Universität in Genf (glücklicherweise von seinen Schülern posthum herausgegeben) wurde der Akzent konsequent von der Diachronie auf Synchronie verlagert. Seine Definition der Sprache als Zeichensystem, die als langage, langue, parole besteht, syntagmatisch und paradigmatisch geordnet, synchronisch und diachronisch existiert und erforscht werden kann, war zu der damaligen Zeit bahnbrechend, dadurch wurde Saussure zum ersten Strukturalisten in der Linguistik. Seitdem wurden linguistische Untersuchungen zunehmend gegenwartsbezogen (synchronisch), auch kontrastiv (nach Zabrocki konfrontativ, 1975).

Der Strukturalismus von de Saussure verbreitete sich wie Lauffeuer aus Genf Europa-weit nach Kopenhagen (Hjemslev) und Prag (Trubetzkoy, Jakobson) und in die USA (Deskriptivismus von Bloomfield, Fries) und wirkte wie eine frische Brise erneuernd in der bislang historisch-diachronisch betriebenen Sprachwissenschaft.

Dies fand seine Auswirkung im FSU: Aus der postulierten direkten ReformMethode (Fremdsprachen auch institutionell direkt zu unterrichten) entwickelte sich sehr bald die audiolinguale Methode, die auf den wissenschaftlichen Grundsätzen der Phonologie in Sprachwissenschaft (seit 1850 wissenschaftliche Disziplin), des Behaviorismus in Psychologie und Strukturalismus in Philosophie und Linguistik fußte.

Diese Unterrichtsform ergab sich aus Beobachtungen des natürlichen Erstspracherwerbs durch Kleinkinder, die ihre erste Sprache (Muttersprache) nur durch Zuhören, Nachsprechen, Ausprobieren und Üben, ohne grammatische Korrekturen erwerben. Dies ist prinzipiell ebenso natürlich wie richtig, nur werden verschiedene kommunikative Fehlleistungen der Kleinkinder, die ihre erste Sprache erwerben von ihrer Umgebung (meistens) nicht bemängelt, sondern mit viel Freude als kreative Neubildungen begrüßt und unterstützt.

Behavioristisch wurde davon ausgegangen, dass das Kleinkind als unbeschriebenes Blatt (tabula rasa) zur Welt kommt, dem man Sprechen durch Zuhören, Nachahmen und (bloßen) Konditionierungsprozess beibringt, also durch die Herstel- 
lung eines dauerhaften Zusammenhangs zwischen äußerem Reiz der Umgebung (Stimulus) und innerer Reaktion des Kindes (Response). Das Kleinkind erwirbt Sprache natürlich, durch ständige Wiederholung von S-R-Serien bis zur Automatisierung, so wie auch motorische Aktivitäten (Gehen, Radfahren, Skifahren) automatisiert erlernt werden. Das Kind sei seiner (aktiven) Umgebung gegenüber passiv, behaupteten Behavioristen.

Dabei stützte man sich auf behavioristische Experimente mit konditionierten Reflexen der amerikanischen Tierpsychologen und Behavioristen, Watson, Thorndike, Skinner, die zur Erkenntnis kamen, Menschen lernen (wie Tiere) durch bloße Wiederholung der S-R-Serien bis zur overlearning-Automatisierung. Es wurde angenommen, dass es erfolgversprechend ist, so zu lernen, wie Tiere lernen und wie Kleinkinder ihre Erstsprache erwerben: durch bloßes Zuhören, lautdiskriminierendes Nachahmen und Auswendiglernen, daher wurde es mim-mem genannt (für mimicry and memorization).

So sollte der audiolinguale FSU prinzipiell auf Zuhören und korrektem Nachsprechen basieren, um auswendig gelernte, bis zum overlearning gedrillte, korrekte Texte (meistens Dialoge) in Situationen des natürlichen Sprachgebrauchs (ebenso nur korrekt) anwenden zu können. Die Unterrichtsprozesse wurden im audiolingualen FSU als symmetrischer Informationsaustausch zwischen Sender (Lehrer/Lerner) und Empfänger (Lerner/Lehrer) aufgefasst; demzufolge wurde angenommen, das fehlerfrei gedrillte Auswendiggelernte reiche auch für eventuelle spontane Ausdrucksmöglichkeiten als Antwort aus.

Amerikanische Linguisten (bspw. Bloomfield, Fries) verfassten auch die dazu notwendigen Lehrmaterialien für den FSU (transformationsreife Drill-Übungssätze in Serien); daher wurde diese Methode „linguistisch“ genannt.

Die audiolinguale Methode fand ihre bekräftigte zwangsmäßige Anwendung, als die USA (im Dezember 1941) dem Zweiten Weltkrieg beigetreten sind. Es ergab sich die dringende Notwendigkeit, fremde Sprachen (hauptsächlich des Fernen Ostens) schnell und near native für militärische Zwecke zu lernen. Durch gedrilltes Nachsprechen und ständiges Üben von Satzmustern (sentence patterns practice) in Dialogsituationen wurden sprachliche Fertigkeiten (skills) in der natürlichen Reihenfolge erworben, d.h., so wie es Kleinkinder tun, wenn sie eine (erste) Sprache erwerben: lautunterscheidendes Zuhören, near native (Nach)Sprechen; ebenso später Lese(verstehe)n und Schreiben.

Unter Einfluss strukturalistischer Sprachkonzeptionen (hauptsächlich der amerikanischen Deskriptivisten Bloomfield, Fries) sowie behavioristischer Lernkonzeptionen (der Tierpsychologen Watson, Thorndike, Skinner (language is a set of habits) auf glottodidaktische Konzeptionen wurde angenommen, Sprache existiert autonom, außerhalb des Menschen (nicht als spezifische menschliche Eigenschaften). Folglich wurde angenommen, der FSU und der Zweitspracherwerb beruhen auf automatisiertem Erwerb bestimmten Wissens, implizit, durch mechanisches Drillen und Aus- 
wendiglernen von Satzstrukturen, auf deren Basis der Lerner weitere Texte (Äußerungen für den realen Gebrauch) selbst bildet. Der eigentliche Ausgangspunkt und die tatsächliche Achse des Unterrichts war die grammatische Progression (und dabei sollte es doch eine Abkehr von der indirekten G-Ü-Methode sein). Dabei handelte es sich hier nur um äußerlich (vom Lehrer) vorgegebenes Wissen und äußerlich vorgegebene Fertigkeiten, die der Lerner auf mechanisch-automatisierende Weise erlernen kann (wie Tiere in der Dressur).

Zwar wurde Grammatik induktiv vermittelt, aber der Unterricht verlief großteils an der Grammatikprogression orientiert: sowohl Lehrwerke als auch Unterrichtsentwürfe waren den grammatischen Zielen untergeordnet, auch Dialoge und Übungen waren mehr an den grammatischen Themen als an der natürlichen, authentischen Spontaneität der Sprache orientiert.

Im audioligualen und audiovisuellen FSU wurden die einzelnen Sprachfertigkeiten additiv, nebeneinander vermittelt; d.h. die Integration der einzelnen Fertigkeiten war unmöglich. Der Weg zum Erreichen der Redegewandtheit sollte durch bloße Assoziationen führen, die durch äußere Impulse gebildet und durch overlearning in den Fertigkeitsbereichen (Hören und Sprechen) gefestigt und automatisiert wurden: vor allem korrektes lautdiskriminierendes Nachsprechen (near native) durch Hören, später sollte Lesen und Schreiben gedrillt werden. Dabei wird Sprache in wirklichen Kommunikations-Situationen integrativ gebraucht, bspw. Hörverstehen gemeinsam mit Schreiben.

Ebenso wurden Lehrmaterial und Lehrprogramm linear, additiv präsentiert: als Beherrschung der Summe der einzelnen Fertigkeiten, die nacheinander, systematisch erlernt wurden (in der manipulativen Etappe) und dann auf Realsituationen übertragen werden sollten (in der Anwendungsetappe) (vgl. Krumm, 2001).

Spontanes Sprechen wurde im audiolingualen Lernen untersagt, nicht zugelassen, weil man glaubte, so Fehlern vorbeugen zu können. Dem ist entgegenzusteuern: kein Mensch lernt, ohne Fehler zu machen; bekanntlich kann nur derjenige keine Fehler machen, der nichts macht. Die damals angestrebte Fehlerlosigkeit des Unterrichts verbannte die spontane Ausdrucksfähigkeit der Schüler. Der Unterschied zur wirklichen Kommunikation ist, in realen Gesprächen konzentriert man sich (meistens) nicht auf Fehler unseres Gesprächsteilnehmers. Es fehlte die Möglichkeit, in wirklichen Situationen frei (also nicht nur nach den vom Lehrer vermittelten Schablonen) zu sprechen. Kaum Beachtung fand anfangs die Entwicklung der Schreibkompetenz im Unterricht sowie die kulturellen und interkulturellen Fragestellungen. Erst nach und nach ergab sich die Notwendigkeit, landeskundliche Informationen bspw. über deutschsprachige Länder zu vermitteln (was später ihren Niederschlag in interkulturellen Ansätzen des FSU fand).

Zwar sollte ins Zentrum des FSU die beabsichtigte Kommunikationsfähigkeit stehen (analog der Natürlichkeit des spontanen Erstspracherwerbs bei Kleinkindern), folglich verschob sich der Schwerpunkt des Unterrichts auf mediale Mündlichkeit, 
also auf Ausspracheschulung (dank der Phonologie wurde die besondere Rolle der Aussprache-Schulung im FSU erkannt, verstärkt durch die aktive Vermittlung der API-Transkription sowie des Vokal-Drei- und Vierecks, was nur positiv zu begrüBen war). Dazu wurden alltägliche Texte v.a. über auswendig gedrillte Dialoge gelernt, der Wortschatz wurde von Alltagsgesprächen in prototypischen Situationen (Äußerungen) geleitet. Verwendet wurden (anschauliche) Visualisierungen, Assoziationen, Nachsprechen und spielerische Aktivitäten (z.B. Rollenspiele in Team-Arbeit), die auch konzeptionelle Mündlichkeit zwischen Ohr und Mund drillen sollten.

Audiolingualer FSU entwickelte sich sehr bald durch den Einsatz von Ton und Bild zur audiovisuellen Methode; er wurde in den 1960er Jahren (auch in Polen) noch durch den Einsatz von Sprachlabors bekräftigt, von denen man sich sehr hohe Lern-Effizienz erhoffte.

Das eigentliche Ziel des audiolingualen FSU sollte die Vorbereitung auf die natürliche, dialogische Kommunikation sein, also das reale, gesellschaftlich effektive Sprachkönnen. Aber es blieb nur beim Versuch, denn zu der damaligen Zeit fehlten theoretische Modelle der Sprachverwendung.

Das damalige linguistische Modell der menschlichen Kommunikation in Polen von L. Zabrocki (1966; 1975), welches sich ausschließlich auf sprachliche Kommunikation konzentrierte (als symmetrischer Transmissionsprozess des Inhalts und der Form zwischen dem Sender und dem Empfänger (mit dem (sprachlichen) Kode, dem Kanal (mündlich, schriftlich), dem Kodieren (mit dem synthetischen Kode des Senders) und Dekodieren (mit dem analytischen Kode des Empfängers), war für den FSU nicht ausreichend. Ebenso ertragreich erwiesen sich die von Jakobson (1960) genannten Funktionen der Sprache im Kommunikationsakt: die expressive, impressive, metasprachliche, phatische und poetische Funktion. Alle stützten sich auf Bühlers Organonmodell des sprachlichen Zeichens (1934: 34) und dessen drei Relationen, des Senders, Empfängers und der Mittleilung als Symbol (zu Gegenständen), als Symptom (in Abhängigkeiten vom Sender) und als Signal (als Appell an den Empfänger). Die chronologischen Meilensteine der weiteren Entwicklung innerhalb der Linguistik und der Glottodidaktik folgten.

7. Die kognitive Wende der 1960er Jahre begann, als Chomsky (1959) die Rezension des Buches von Skinner (1957) schrieb, was zur Abwendung in der Psychologie vom Behaviorismus führte. In der mentalistischen Sprachtheorie von Chomsky (1965) kommt der Universalgrammatik und der Kreativität (besonders im Erstspracherwerb des Kleinkindes) als angeborene Fähigkeit und Voraussetzung des Menschen, Sprachen zu erwerben, eine bedeutende Rolle zu. Daneben entwickelte Chomsky die Konzeption der sprachlichen Kompetenz und Performanz. In seiner Grammatiktheorie wurde Kompetenz eines idealen Sprechers/Hörers zum grundlegenden sprachlichen Vermögen, das im Sinne der Universalgrammatik als angebo- 
rene Fähigkeit zur Erzeugung von grammatisch korrekten Sätzen dient (und nach Chomsky der eigentliche Gegenstand der Linguistik sein soll).

Chomsky interessierte sich für die Sprachperformanz, also den tatsächlichen Sprachgebrauch nicht, weil dieser mit Fehlern belastet ist (daher kein grammatisches Ideal darstellt); daher blieb der pragmatische Gebrauchsaspekt der Sprache von Chomsky unbeachtet.

Obwohl sich Chomsky nicht zum FSU äußerte, wirkte sein Gedankengut auf den FSU inspirierend. Grucza (2007: 377) nennt Chomskys Sprachtheorie den entscheidenden Schritt in der Entwicklung der Linguistik, weil so der Schwerpunkt der Linguistik von der Sprache als einem Struktursystem zur Rekonstruktion der Sprache als Regelwerk, das die Menschen befähigt, Texte zu produzieren - auf den Menschen (als Produzenten und Empfänger, also Sprachbenutzer) verschoben wurde. Schwarz (2008: 9) nennt Chomskys Abwendung vom Behaviorismus die kognitive Wende.

Auch wenn das Modell der Sprachkompetenz von Chomsky ein sehr abstraktes linguistisches Modell war, zu abstrakt, um im FSU Anwendung zu finden, wurde es (a contrario) zum Ansporn für theoretische Überlegungen zu der auf Sprachgebrauch gerichtete Linguistik (ebenso für den FSU).

8. Die kommunikative Wende der 1970er Jahre entwickelte sich als Gegenreaktion zu Chomskys Hauptinteresse für die Sprachkompetenz und Vernachlässigung des Sprachgebrauchs. Es entwickelten sich zu Beginn der 1970er Jahre weltweit linguistische Richtungen, die auf wirklichen Sprachgebrauch abzielen, wie Pragmalinguistik, Soziolinguistik, Ethnolinguistik. So kam es zur sog. kommunikativen Wende in der Linguistik, folglich auch im FSU. Austin (1962) und Searle (1969) entwickelten die Konzeption der Sprechakttheorie, dessen Ziel es war, auf authentischen wirklichen, situationsadäquaten, handlungsorientierten Sprachgebrauch hinzuweisen. Sprache wird als soziale Handlungen aufgefasst; jedes Sprechen ist aktives Tun, gesellschaftlich geprägtes, intentional und zielorientiertes Handeln. In den Mittelpunkt des linguistischen Interesses werden folglich Sprechakte gestellt, bspw. jn empfangen, begrïßen, (Begrüßung), nicht grammatisch korrekte oder unkorrekte Wörter und Sätze.

Hymes (1972) entwickelte die Konzeption der kommunikativen Kompetenz (gegenüber Chomskys Sprachkompetenz und als Aufwertung der von Chomsky vernachlässigten Performanz). Ausgangspunkt sind dabei Relationen zwischen dem sprachlichen Zeichen und dessen Benutzern im Kommunikationsprozess: also das, was man mit Sprache machen kann (und nicht (nur) grammatische Korrektheit). Über die kommunikative Kompetenz der Gesprächsteilnehmer entscheiden gesellschaftliche und kulturdeterminierte sprachliche und nichtsprachliche Kommunikationsmittel, die situationsadäquat und intendiert eingesetzt werden.

In Deutschland entwickelte Habermas (1971) die deduktive Theorie der kommunikativen Kompetenz, die auf gesellschaftliche zwischenmenschliche Relationen 
und auf subjektive Determinanten des Sprechens, wie die Möglichkeiten, Wissen, Können und Absichten der einzelnen Kommunikationsteilnehmer ausgerichtet ist.

Die linguistischen Einflüsse der Sprechakttheorie hatten gravierenden Einfluss auf die weitere Entwicklung innerhalb der Linguistik sowie auf die Priorität der Mündlichkeit im FSU. Für den FSU bedeutete die kommunikative Umwandlung kommunikative Kompetenz im FSU zu vermitteln: die Lerner sollten im DaF lernen, mündlich zu handeln (nach Austin „Dinge mit Worten tun“), d.h. in Partner-, Gruppenarbeit, Rollenspielen gesellschaftlich adäquat, authentisch und spontan reagieren.

Der FSU wurde kommunikativ und handlungsorientiert: Lernen wurde nicht mehr (behavioristisch) als Verhaltensänderung durch natürlichen oder konditionierten Reiz mit dem Lerner als passives Unterrichtsobjekt aufgefasst, sondern ins Zentrum des Unterrichtsgeschehens wurde der Sprach-produzierende und -empfangende Mensch mit seinen subjektiven Gegebenheiten (Wissen, Können, Absichten, Motivationen) gestellt, die den wirklichen Kultur-determinierten Sprachgebrauch ermöglichen.

Der Lerner wurde nicht mehr als passives Unterrichtsobjekt gesehen, sondern als aktives Unterrichtssubjekt, als verantwortungsbewusster Teilnehmer im gesellschaftlichen schulischen Interaktionsprozess (Lernerzentriertheit des FSU); der Lehrer wurde zum Helfer, Moderator, Erzieher, Wissensvermittler, der seinen Lernern Mengen von Handlungsmitteln für das Kommunizieren in der fremden Sprache optimal bereit stellt (nicht mehr Allwisser); es änderten sich die Sozialformen im Unterricht, der zunehmend auf Partnerarbeit, Teamarbeit, Projekten, Rollenspielen basierte (nicht mehr nur auf Frontalunterricht). Man begann von Bewusstheit und Autonomie im Lern- und Unterrichtsprozessen zu sprechen.

Das Ziel des FSU wurde nun auf Sprechakte, Sprechhandlungen gelenkt, also darauf, was und wie mit Sprache gemacht werden kann (nicht mehr auf bloße lexikalische und grammatische Sprache), wie man mit Sprache praktisch und zielgerecht umgehen (also kommunizieren) kann. Das Fremdsprachenlernen wurde zunehmend als handlungsorientierter Vorgang gesehen, in dem fremde Sprache im authentischen Gebrauch, sozial angemessen, geübt wird.

Mit der Entwicklung der kommunikativem Kompetenz im FSU kam es zunehmend zur Ausgrenzung der Grammatik und zur Entwicklung der (konzeptuellen) Mündlichkeit, die Vorrang vor Schriftlichkeit hat.

Der handlungsorientierte FSU erforderte neue Lehrpläne und Lehrwerke, wie Neuners et al. 1979 Deutsch aktiv (für DaF), als das bahnbrechende Beispiel für Umorientierung der Lehrwerke auf kommunikative Kompetenz für den Unterricht $\mathrm{DaF}$, also auf Sprachhandlungen und Sprachfunktionen (anstatt auf Wortartengrammatik).

9. Der pragmatisch-funktionale Ansatz entwickelte sich in den 1980er Jahren, gestützt intensiviert und erweitert auf kommunikativem Handeln: sprechen mit 
(interaktives Teilnehmen an gesellschaftlichen Relationen), und sprechen über (neue Textsorten werden verwendet, wichtig wird Erzählen, ebenso verschiedene (altersbedingte und dialektale) Sprachvarietäten - konzentriert auf Text-Verstehen und intendiertem Text-Produzieren von Äußerungen. Zunehmend kommt es zur Ausgrenzung der Grammatik gegenüber der authentischen mündlichen Kommunikation; Mündlichkeit hat Vorrang vor Schriftlichkeit.

Entwicklungen in der Linguistik der 1980er Jahre beruhten auf weiteren Forschungen der gesprochenen Sprache, die zur Unterscheidung zwischen medialer Mündlichkeit (Ausspracheschulung) und konzeptioneller Mündlichkeit (Gesprächsschulung) führten (Hirschfeld, Rösler und Schramm, 2016). Diese Unterscheidung erstreckte sich auch auf (mediale und konzeptionelle) Schriftlichkeit. All das fand seinen Niederschlag im FSU, der weiterhin Redemittel-orientiert verlief; Sprechhandlungen wie Erzählen, Beschreiben, Berichten, Erklären, Argumentieren wurden integrativ in ihrem funktionalen Zusammenhang (hauptsächlich mündlich) trainiert.

Dazu wurden Lehr-Lern-materialien auf die Auswahl und Sequenzierung von satz- und textbezogenen Grammatikphänomenen konzentriert. Dem folgten Lehrmaterialien des Europarats für DaF von Baldegger et al. (1981) Kontaktschwelle für DaF.

10. In den 1990er Jahren fanden Sprachlernstrategien und Kommunikationsstrategien ihren bewussten Einsatz sowohl in der Fremdsprachendidaktik als auch im alltäglichen FSU: Approximationen und nichtsprachliche Mittel als Ersatz für fehlende (sprachliche, kultubasierte) Ausdrucksmittel werden explizit vermittelt.

11. Im Jahre 2001 wurde der Gemeinsame europäischer Referenzrahmen für Sprachen: lernen, lehren, beurteilen als normativer Wegweiser für institutionelles Fremdsprachenlernen herausgegeben. Der Titel weist darauf hin, dass die Primärposition dem Lernen zukommt, dem Unterrichten und ständiges Bewerten (lerner- und lehrerseits) folgt. Im Vordergrund stehen Redemittel für (monologische) Produktion und Rezeption sowie für (mündliche und schriftliche) Interaktion (also nicht mehr Entwicklung der vier Fertigkeiten: Sprechen, Hören, Lesen, Schreiben).

Zusammenfassend ist festzuhalten, im FSU soll kulturdeterminierte Sprache, in ihren (geografischen und sozialen) Varietäten, begleitet von nichtsprachlichen kommunikativen Mitteln für monologische Perzeption und Produktion sowie ausgewogen integrative (sowohl mediale als auch konzeptionelle) Mündlichkeit und Schriftlichkeit für gesellschaftlich intendierte, situationsadäquate Interaktion für den allgemeinsprachlichen Gebrauch und/oder für den beruflichen und FachsprachenGebrauch, vermittelt werden. All das unter Berücksichtigung von Mehrsprachigkeitsdidaktik (und für DaF die Position Deutsch nach Englisch als Fremdsprache, DnEaF) im Gefüge der Tertiärsprachendidaktik, mit dem Hinweis, die Erstsprache (Muttersprache) unter dem Trans-Aspekt der Sprachkontakte (Puppel, 2007) nicht zu vernachlässigen (Wiertlewska, 2010). 
Und trotzdem Englisch momentan als Globalsprache im FSU die am meisten unterrichtete Sprache ist, wird DaF in Polen weltweit am meisten unterrichtet (Földes, 2016).

In den Jahrzehnte lang dauernden kognitivistisch-konstruktivistische Bemühungen um den kulturdeterminierten kommunikativen FSU, sollte der Unterricht sowohl auf mediale Mündlichkeit und Schriftlichkeit, als auch auf konzeptionelle Mündlichkeit und Schriftlichkeit konzentriert sein. Dies ist nur bei der individualisierten Zusammenarbeit des autonomen (paido- und logotropen) Lehrers mit dem autonomen Lerner, der bewusst und aktiv mitverantwortlich für den FSU ist. Dazu gehört u.a., eine bewusste Kenntnis von Lernstrategien für den Unterricht: der Lerner muss das Lernen bewusst erlernen (Kaniewska, 2016: 6).

\section{Bibliografie}

Austin, J.L. 1962. How to do things with words. Oxford: Clarendon Press.

Bühler, K. 1934. Die Sprachtheorie. Die Darstellungsfunktion der Sprache. Jena: G. Fischer.

Baldegger, M., Müller, M. und G. Schneider. 1981. Kontaktschwelle Deutsch als Fremdsprache. Berlin/München: Langenscheidt.

Chomsky, N. 1959. „A review of B.F.Skinner's 1957 verbal behavior”. Language 1. $26-54$.

Chomsky, N. 1965. Aspects of the theory of syntax. Cambridge, Mass: MIT Press.

Földes, C. 2016. „Position und Entwicklungsdynamik des Deutschen als Fremdsprache international im Blick der angewandten Linguistik". Glottodidactica 43.2. 13-38.

Grucza, F. 1988. „O filologii, neofilologii i kształceniu nauczycieli języków obcych”. In: Grucza, F. (Hrsg.). Problemy kształcenia nauczycieli języków obcych. Warszawa: Wydawnictwa Uniwersytetu Warszawskiego. 17-67.

Grucza, F. 2007. „Über den Status der Angewandten Linguistik”. In: Grucza, F. (Hrsg.). Lingwistyka stosowana. Historia - zadania - osiagnięcia. Warszawa: Euro-Edukacja. 349-370.

Habermas, J. 1971. „Vorbereitende Bemerkungen zu einer Theorie der Kommunikativen Kompetenz”. In: Habermas, J. und N. Luhmann (Hrsg.). Theorie der Gesellschaft oder Sozialtechnologie. Frankfurt am Main: Surkamp. 101-141.

Hymes, D. 1972. „On communicative competence”. In: Pride, J.B. und J. Holmes (Hrsg.). Sociolinguistics. Harmondsworth: Penguin. 269-293.

Hirschfeld, U., Rösler, D. und K. Schramm. 2016. „Facetten der Mündlichkeit im DaF-Unterricht. Zur Einführung in den Themenschwerpunkt". DaF 3. 131-134.

Jakobson, R. und M. Halle. 1960. Grundfragen der Sprache. Berlin: Akademie-Verlag.

Kaniewska, B. 2016. „Najważniejsze to nauczyć się uczyć”. Życie Uniwersyteckie 11. 6-7.

Krumm, H.-J. 2001. „Die sprachlichen Fertigkeiten: isoliert-kombinieret-integriert“. Fremdsprache Deutsch 24. 5-12.

Neuner, G., Schmidt, R. und H. Wilm. 1979. Deutsch aktiv. Arbeitsbuch. Berlin: Langenscheidt.

Puppel, S. 2007. „Interlingwalizm czy translingwalizm? Interkomunikacja czy transkomunikacja? Uwagi w kontekście współistnienia języków naturalnych w kontekście globalnej wspólnoty kulturowojęzykowo-komunikacyjnej”. In: Puppel, S. (Hrsg.). Społeczeństwo - kultura - język. W stronę interakcyjnej architektury komunikacji. Poznań: Katedra Ekokomunikacji UAM. 79-94.

Rösler, D. 2016. „Nähe und Distanz zur Mündlichkeit in der fremdsprachendidaktischen Diskussion. Versuch einer Annäherung“. DaF 3. 135-149. 
Searle, J.R. 1969. Speech acts. Cambridge: Cambridge University Press.

Schwarz, M. 2008. Einführung in die kognitive Linguistik. Tübingen: Francke.

Trim, J., North, B., Coste, D. und J. Sheils. 2001. Gemeinsamer europäischer Referenzrahmen für Sprachen: lernen, lehren, beurteilen. Straßburg: Europarat / Berlin/München: Langenscheidt KG.

Viëtor, W. 1882. Der Sprachunterricht muss umkehren! Ein Beitrag zur Überbürdungsfrage von Quosque Tandem. Heilbronn: Gebr. Henningen.

Wiertlewska, J. 2010, Ekolingwistyczne podejście do nauczania języków obcych na przykładzie języka angielskiego - wyzwaniem dla glottodydaktyki XXI wieku. Toruń: UMK.

Zabrocki, L. 1962. „Reorganizacja studiów neofilologicznych”. Życie Szkoły Wyższej 10.5. 43-51.

Zabrocki, L. 1966. „Kodematische Grundlagen der Theorie des Fremdsprachenunetrrichts“. Glottodidactica 1. 3-42.

Zabrocki, L. 1967. „Profil absolwenta neofilologii”. Życie Szkoty Wyższej 15.9. 54-64.

Zabrocki, L. 1975. Kybernetische Modelle der sprachlichen Kommunikation. Wrocław: Ossolineum. 